\title{
Dark matter direct search sensitivity of the PandaX-4T experiment
}

\author{
Hongguang ZHANG,${ }^{1}$ Abdusalam ABDUKERIM,${ }^{2}$ Xun CHEN, ${ }^{1}$ Yunhua CHEN,${ }^{3}$ Xiangyi CUI, ${ }^{1}$ Binbin \\ DONG, ${ }^{1}$ Deqing FAnG,${ }^{4}$ Changbo FU, ${ }^{1}$ Karl GIBOnI, ${ }^{1}$ Franco GIULIANI, ${ }^{1}$ Linhui GU, ${ }^{1}$ Xuyuan GUO,${ }^{3}$ \\ Zhifan GUO,${ }^{5}$ Ke HAN,${ }^{1}$ Changda HE, ${ }^{1}$ Shengming HE, ${ }^{3}$ Di HUANG,${ }^{1}$ Xingtao HUANG,${ }^{6}$ Zhou HUANG,${ }^{1}$ \\ Peng JI, ${ }^{7}$ Xiangdong JI, ${ }^{1,8, \text { | }}$ Yonglin JU, ${ }^{5}$ Shaoli LI, ${ }^{1}$ Yao LI, ${ }^{1}$ Heng LIN,${ }^{1}$ Huaxuan LIU, ${ }^{5}$ Jianglai LIU, $, 1,8$ \\ Yugang MA, ${ }^{4}$ Yajun MAO, ${ }^{9}$ Kaixiang NI, ${ }^{1}$ Jinhua NING, ${ }^{3}$ Xiangxiang REN, ${ }^{1}$ Fang SHI, ${ }^{1}$ Andi TAN, ${ }^{10}$ \\ Anqing WANG,${ }^{6}$ Cheng WANG,${ }^{5}$ Hongwei WANG, ${ }^{4}$ Meng WANG,${ }^{6}$ Qiuhong WANG,${ }^{4}$ Siguang WANG ${ }^{9}$ \\ Xiuli WANG, ${ }^{5}$ Xuming WANG, ${ }^{1}$ Zhou WANG,${ }^{5}$ Mengmeng WU, ${ }^{7}$ Shiyong WU, ${ }^{3}$ Jingkai XIA, ${ }^{1}$ Mengjiao \\ XIAO,${ }^{10,11}$ Pengwei XIE, ${ }^{8}$ Binbin YAN,${ }^{6}$ Jijun YANG, ${ }^{1}$ Yong YANG, ${ }^{1}$ Chunxu YU, ${ }^{7}$ Jumin YUAN,${ }^{6}$ Jianfeng \\ YUE,${ }^{3}$ Dan ZHANG, ${ }^{10}$ Tao ZHANG,${ }^{1}$ Li ZHAO,${ }^{1}$ Jifang ZHOU, ${ }^{3}$ Ning ZHOU,, 1 , and Xiaopeng ZHOU ${ }^{9}$ \\ ${ }^{1}$ INPAC and School of Physics and Astronomy, Shanghai Jiao Tong University, \\ Shanghai Laboratory for Particle Physics and Cosmology, Shanghai 200240, China \\ ${ }^{2}$ School of Physics and Technology, Xinjiang University, Urumqi 830046, China \\ ${ }^{3}$ Yalong River Hydropower Development Company, \\ Ltd., 288 Shuanglin Road, Chengdu 610051, China \\ ${ }^{4}$ Shanghai Institute of Applied Physics, Chinese Academy of Sciences, Shanghai 201800, China \\ ${ }^{5}$ School of Mechanical Engineering, Shanghai Jiao Tong University, Shanghai 200240, China \\ ${ }^{6}$ School of Physics and Key Laboratory of Particle Physics and Particle \\ Irradiation (MOE), Shandong University, Jinan 250100, China \\ ${ }^{7}$ School of Physics, Nankai University, Tianjin 300071, China \\ ${ }^{8}$ Tsung-Dao Lee Institute, Shanghai 200240, China \\ ${ }^{9}$ School of Physics, Peking University, Beijing 100871, China \\ ${ }^{10}$ Department of Physics, University of Maryland, College Park, Maryland 20742, USA \\ ${ }^{11}$ Center of High Energy Physics, Peking University, Beijing 100871, China
}

\begin{abstract}
The PandaX-4T experiment, a four-ton scale dark matter direct detection experiment, is being planned at the China Jinping Underground Laboratory. In this paper we present a simulation study of the expected background in this experiment. In a 2.8-ton fiducial mass and the signal region between 1 to $10 \mathrm{keV}$ electron equivalent energy, the total electron recoil background is found to be $4.9 \cdot 10^{-5}(\mathrm{~kg} \cdot \text { day } \cdot \mathrm{keV})^{-1}$. The nuclear recoil background in the same region is $2.8 \cdot 10^{-7}(\mathrm{~kg} \cdot$ day . $\mathrm{keV})^{-1}$. With an exposure of 5.6 ton-years, the sensitivity of PandaX-4T could reach a minimum spin-independent dark matter-nucleon cross section of $6 \cdot 10^{-48} \mathrm{~cm}^{2}$ at a dark matter mass of $40 \mathrm{GeV} / c^{2}$.
\end{abstract}

\section{INTRODUCTION}

Precise astrophysical and cosmological observations indicate that most of the universe is composed of the dark matter and dark energy [1, 2]. In the standard model of cosmology, the dark matter contributes up to $26.8 \%$ of the total content of the universe [3]. Near our solar system, the local dark matter density is estimated to be 0.3 $\mathrm{GeV} / \mathrm{cm}^{3}$ [4. One of the most favored dark matter particle candidates are the so-called Weakly Interacting Massive Particles (WIMPs) predicted in many theories beyond the Standard Model of particle physics [5]. Theories also predict a feeble interaction between the WIMPs and the normal matter, so when WIMPs pass through a detector on Earth, they could produce detectable low-energy nuclear recoil signals. This is the principle of dark matter direct detection [6].

Dual-phase xenon detectors are currently leading in the search for WIMPs with mass ranging from a few $\mathrm{GeV} / c^{2}$ to several $\mathrm{TeV} / \mathrm{c}^{2}$ [7. In a dual-phase xenon detector, the WIMP-induced nuclear recoil (NR) events have two signals: a prompt $S 1$ signal due to scintillation photons in the liquid xenon and a delayed $S 2$ signal from ionization electrons, which drift to the liquid surface and then produce electroluminescence photons in the xenon gas. The $S 1$ and $S 2$ signals are collected by two arrays of photomultiplier tubes (PMTs) on the top and bottom of the detector. The WIMP-nucleon scattering vertex in three-dimensions can be reconstructed based on the photon pattern on the PMT arrays and the time difference between the $S 1$ and $S 2$ signals. This type of detector is also commonly referred to as the time projection chamber (TPC). Most of the background events in the detector are from electron recoils (ER). These events have different proportion in $S 1$ and $S 2$ signals compared to the NR events, so a large fraction can be identified and removed. During the past years, dual phase xenon experiments LUX[8, XENON1T 9 ] and PandaXII [10, 11] have been producing new data continuously and pushed the constraints on the WIMP-nucleon scattering cross section down to a lowest level of $10^{-46} \mathrm{~cm}^{2}$ at a dark matter mass around $50 \mathrm{GeV} / c^{2}$. In this paper, we shall 
discuss the upgrade of PandaX-II, the 4-ton scale PandaX-4T experiment, and evaluate its potential sensitivity to WIMP detection.

The rest of this paper is organized as follows. Sec. describes the PandaX-4T experiment and detector design. The ER and NR backgrounds are discussed in Sec. and Sec. , respectively, followed by a summary in Sec. . The WIMP sensitivity is presented in Sec. , followed by a conclusion in Sec. .

\section{THE PANDAX-4T EXPERIMENT}

The PandaX-4T experiment will be located in the B2 experimental hall in the second phase of China Jinping underground Laboratory (CJPL-II). With a 2400-m marble overburden, the cosmic ray flux in CJPL is reduced to a negligible level $\left(2 \cdot 10^{-10} \mathrm{~cm}^{-2} \mathrm{~s}^{-1}\right)$. In addition, the PandaX-4T detector will be placed in the center of $13 \mathrm{~m}$ tall, $10 \mathrm{~m}$ diameter water tank (Fig. 1) containing ultrapure water to suppress the external gammas and neutrons from the laboratory environment to a negligible level.

To ensure an ultra-clean background for the PandaX-4T experiment, materials with a low level of radioactivity are selected for the detector construction. The central cylindrical TPC has a transverse diameter of $1.2 \mathrm{~m}$ and a height of $1.3 \mathrm{~m}$. It is contained inside a cryostat with an outer vessel and inner vessel constructed with low background stainless steel (SS). The inner vessel which holds the liquid xenon $(-100 \mathrm{C})$ is composed of a barrel and two domes. The barrel is approximately 1.8 meters in height and 1.3 meter in diameter, with a weight of about 0.5 ton. The bottom dome is welded to the barrel. The top dome has a SS flange which seals to the top of the barrel, with a total weight of 0.5 ton. The outer vessel, with a gap of $12.5 \mathrm{~cm}$ to the inner vessel, provides a vacuum thermal insulation for the inner vessel, and the total weight is approximately 1.5 tons. The total mass of liquid xenon is approximately 6 tons, and the liquid xenon contained in the sensitive volume (TPC) is 4 tons (see Figure 1).
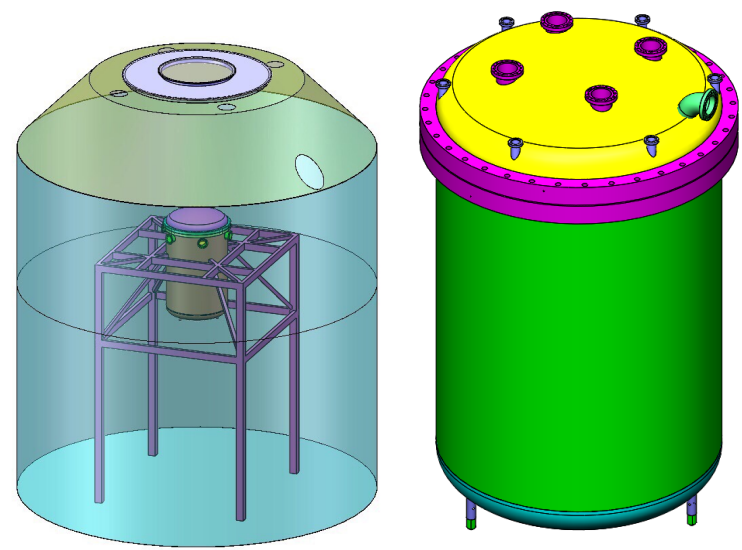

FIG. 1: Left: The ultrapure water shield for PandaX-4T. Right: the inner cryostat.

The TPC consists of two $20 \mathrm{~mm}$ thick copper plates and 24 pieces of $6 \mathrm{~mm}$ thick interlocking polytetrafluoroethylene (PTFE) panels, which laterally form an approximately cylindrical structure with a diameter of 1.2 meters, as shown in Figure 2, Four highly transparent electrodes (from top to bottom: anode, gate, cathode and screening) are designed inside the TPC to produce needed electric fields. The electrodes are made of SS rings with $0.2 \mathrm{~mm}$ SS mesh fixed on them. The cathode is placed $100 \mathrm{~mm}$ above the bottom copper plate, and the gate and anode electrodes are separated by $10 \mathrm{~mm}$ with liquid surface in the middle. The drift distance between the gate and cathode electrodes is 1.2 meters. The design drift field is $400 \mathrm{~V} / \mathrm{cm}$ in the liquid xenon, and the amplification field in the gas xenon is $6000 \mathrm{~V} / \mathrm{cm}$. Directly on top of the bottom copper plate, there is a screening electrode which is grounded. In addition, approximately 60 copper shaping rings surrounding the TPC are designed to maintain an uniform vertical drift field inside the TPC, especially close to the PTFE wall. The total mass of the shaping rings is approximately $45 \mathrm{~kg}$. 24 pieces of PTFE pillars are designed to connect the top and bottom copper plates and to hold the electrodes and shaping rings. A layer of PTFE is attached to the inner wall of the cryostat, and the liquid xenon between the TPC and the wall is a highly reflective and optically separated region used as a veto compartment.

For the signal photon collection, the low background 3-inch PMTs Hamamatsu R11410-23 are fixed onto the top and bottom copper plates with photocathodes facing inward. There are 169 top PMTs placed in a concentric circular pattern, approximately $50 \mathrm{~mm}$ above the liquid surface, and 199 bottom PMTs in a compact hexagonal structure 


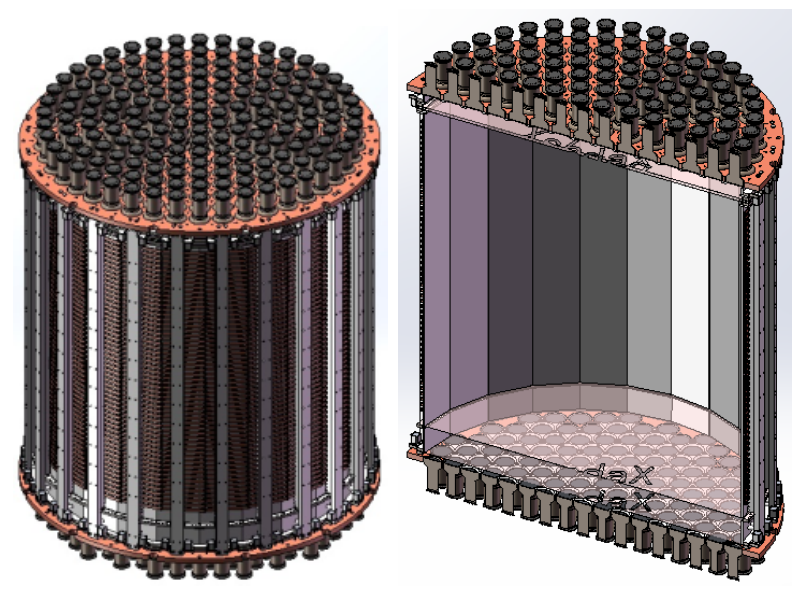

FIG. 2: The diagram of the PandaX-4T TPC.

TABLE I: Material Radioactivity for PandaX-4T: The radioactivity values of PTFE and R11410 PMT components (window and stem) are took from the Xenon1T experiment, while others are the same as the PandaX-II experiment.

\begin{tabular}{lccccccc}
\hline \hline & \multicolumn{3}{c}{ Radioactivity: mBq/unit } & & & \\
\hline Material & ${ }^{60} \mathrm{Co}$ & ${ }^{40} \mathrm{~K}$ & ${ }^{137} \mathrm{Cs}$ & ${ }^{238} \mathrm{U}_{\mathrm{e}}$ & ${ }^{238} \mathrm{U}_{1}$ & ${ }^{235} \mathrm{U}^{232} \mathrm{Th}_{\mathrm{e}}$ & ${ }^{232} \mathrm{Th}_{1}$ \\
\hline SS 3000 kg & 1.03 & 13.95 & 2.36 & 1.7 & 1.7 & 2.43 & 2.74 \\
\hline Copper 200 kg & 0.2 & 4 & 0.16 & 0.38 & 0.38 & 0.86 & 0.51 \\
\hline PTFE 200 kg (XENON1T) & 0.027 & 0.34 & 0.17 & 0.25 & 0.12 & 0.01 & 0.04 \\
\hline R11410 PMT 368 pics & 3.5 & 13 & 0.3 & 0.94 & 0.94 & 1.17 & 0.07 \\
\hline R11410 Window(Quartz) 368 pics (XENON1T) & 0.01 & 0.02 & 0.01 & 1.2 & 0.07 & 0.02 & 0.03 \\
\hline R11410 Stem(Al $\mathrm{O}_{3}$ ) 368 pics (XENON1T) & 0.02 & 1.1 & 0.02 & 2.4 & 0.26 & 0.11 & 0.23 \\
\hline R8520 PMT 144 pics & 0.75 & 8.15 & 0.17 & 0.25 & 0.25 & 0.11 & 0.46 \\
\hline
\end{tabular}

placed approximately 1.3 meter below the liquid surface. The space between the PMT photocathodes is covered with PTFE for better photon collection. Two circles of 1-inch Hamamatsu R8520-406 PMTs are placed at the top and bottom of the veto compartment to collect photons produced by peripheral background.

\section{BACKGROUND ESTIMATION}

The WIMP signals are expected to be single scatterering events, uniformly distributed in the target volume. WIMPnucleus recoil signals yield a typical energy of tens of $\mathrm{keV}$, and a nominal WIMP search window is between 1 to 10 $\mathrm{keV}$ electron equivalent energy. Due to the self-shielding from the liquid xenon, the radioactive background from the detector materials will be located close to the edge of the target, and a large fraction can also be removed due to multiple scattering vertices. The other backgrounds due to trace amount of radioactive noble gases, e.g. ${ }^{85} \mathrm{Kr},{ }^{222} \mathrm{Rn}$ and ${ }^{136} \mathrm{Xe}$, would diffuse uniformly in the target, so would the events due to solar neutrinos.

The radioactivities of all materials used in the detector construction were measured in a high purity Germanium (HPGe) gamma-ray detector located at CJPL, and are inputs to the background estimation. For the PTFE materials, since the natural radioactivities are too low to be measured by HPGe, we adopted values from XENON1T [12] which were measured after neutron activation. For other materials, when only upper limits are available from the measurement, we take them as the central values for conservative background estimation. The radioactivities of the PMT were measured by us as a single component. For the ER background estimation for the PMTs, we took the measured values or upper limits and assumed that they originate from the quartz windows. But the corresponding NR background was computed component by component (e.g. quartz window, ceramic stem) based on the measured values from XENON1T. The radioactivity of the materials is summarized in Table 1 .

A customized Geant4 [13] (ver. 10.2p02) simulation program with the above geometry implemented, BambooMC, is used to simulate the decay of the radioactive isotopes and their energy depositions inside the liquid xenon. For each deposition, information of the deposited energy, position, time and interaction type are recorded. A post-simulation reconstruction algorithm is developed to cluster the neighboring depositions into individually measurable scatters so that the comparison between the MC simulation and data is possible. Only scatters with an energy exceeding the 
threshold of PandaX-II (1 keV electron equivalent energy) are considered.

\section{ER Background}

All sources of ER background are considered in this section, which include the radioactive contamination from detector materials, intrinsic radioactive isotopes such as ${ }^{85} \mathrm{Kr},{ }^{222} \mathrm{Rn}$, and ${ }^{136} \mathrm{Xe}$ in liquid xenon, and neutrinos from the sun. The rate of the background is calculated between 1 to $10 \mathrm{keV}_{e e}$, with a 2.8-ton fiducial cut.

As the detector is shielded by the ultrapure water shield, the laboratory background from the concrete wall, water tank, and others materials in the laboratory is suppressed to a negligible level.

In the detector simulation, the materials we considered include the SS, copper, PTFE and the PMTs using the radioactivity levels from Table I. For each isotope, $10^{8}$ decays are generated uniformly in the component volume. Within the dark matter signal selection windows (energy window, fiducial volume and single scattering), we count the survival events and convert to the background rate. The ER background distribution inside the TPC is shown in Figure 3 .

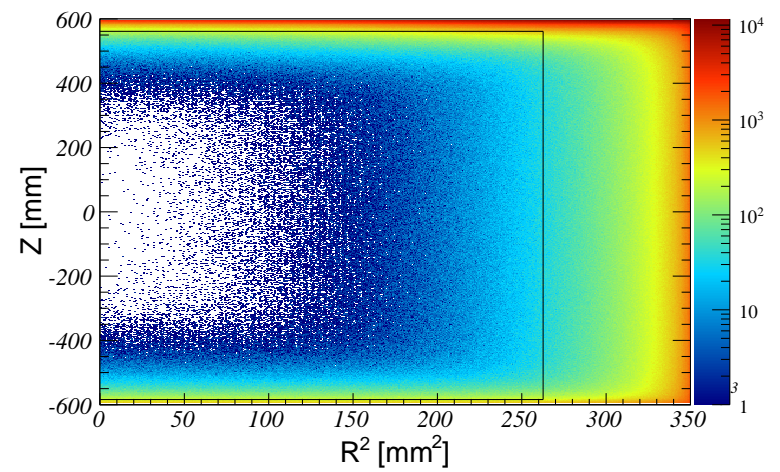

FIG. 3: The distribution of the simulated ER background events after the dark matter signal selection cuts in the TPC.

For the ER background, some single scatter events in the TPC may also deposit energy in the veto compartment, and the veto PMTs provide additional background suppression ability. We assume that the veto compartment triggers at an energy deposition larger than $150 \mathrm{keV}$, a conservative value adopted from PandaX-II experiment. When such a veto is applied to the ER background, we find approximately $50 \%$ of the ER background from the TPC materials can be rejected.

The ER rate spectrum is shown in Figure 4. from which the background rates of various components can be determined. The background rate is measured in $\mathrm{mDRU}\left(=10^{-3}\right.$ events $/$ day $\left./ \mathrm{kg} / \mathrm{keV}\right)$. Various isotopes' contributions from the PMTs and the SS vessels are shown in Figure 5 . We find most of the background comes from ${ }^{232} \mathrm{Th},{ }^{238} \mathrm{U}$ and ${ }^{60} \mathrm{Co}$.

The total ER background from the detector materials is estimated to be $0.021 \mathrm{mDRU}$, as summarized in Table II. It indicates that the largest contribution comes from the SS vessels due to their large mass surrounding the TPC. The second largest contribution is from the PMT arrays, as they are very close to the target volume. A $20 \%$ uncertainty is adopted based on the HPGe counting uncertainties.

TABLE II: ER background from detector materials for $E_{e e}$ within $(1 \mathrm{keV}, 10 \mathrm{keV})$.

\begin{tabular}{cc}
\hline \hline \multicolumn{2}{c}{ ER Background from materials } \\
\hline Source & ER in mDRU \\
\hline Inner vessel & $0.0064 \pm 0.0013$ \\
\hline Outer vessel & $0.0088 \pm 0.0018$ \\
\hline PMT & $0.0045 \pm 0.0009$ \\
\hline PTFE & $0.0005 \pm 0.0001$ \\
\hline Copper & $0.0008 \pm 0.0002$ \\
\hline Total material & $0.0210 \pm 0.0042$ \\
\hline
\end{tabular}




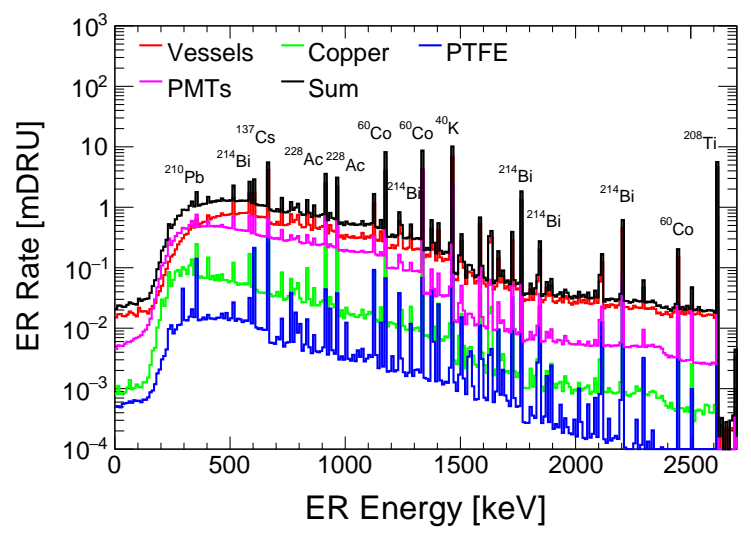

FIG. 4: The simulated ER energy spectra from detector materials and various components (PTFE, PMTs, SS vessels and copper).
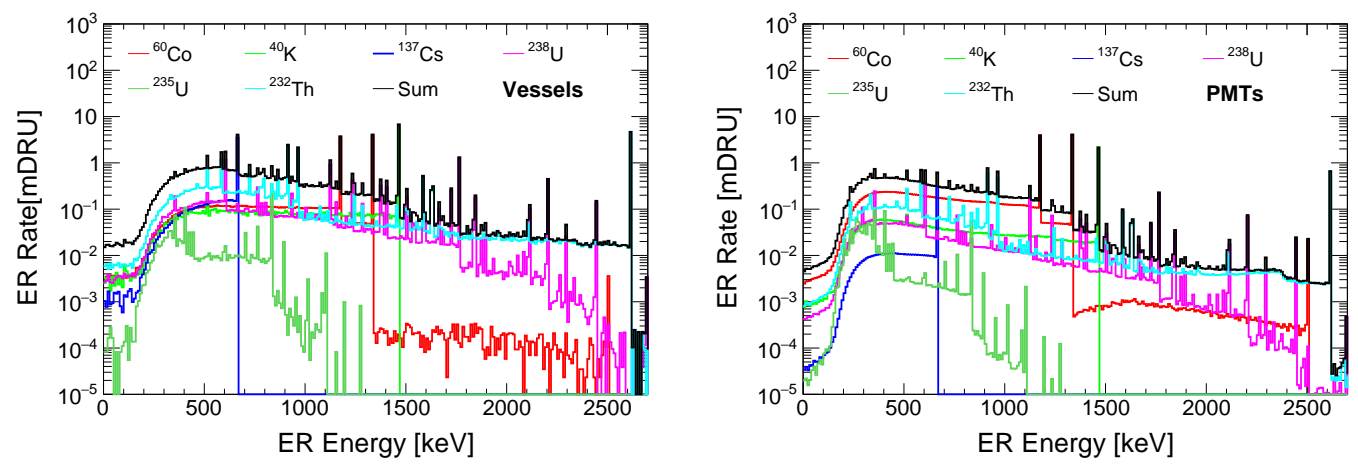

FIG. 5: Electron recoil background from isotopes $\left({ }^{60} \mathrm{Co},{ }^{40} \mathrm{~K},{ }^{137} \mathrm{Cs},{ }^{235} \mathrm{U},{ }^{238} \mathrm{U}\right.$ and $\left.{ }^{232} \mathrm{Th}\right)$. Results for the vessels and PMTs are plotted separately.

For internal background inside the liquid xenon, we first considered ${ }^{85} \mathrm{Kr} .{ }^{85} \mathrm{Kr} \beta$-decays with an end-point energy $687 \mathrm{keV}$ and a half-life of 10.76 years. The abundance of ${ }^{85} \mathrm{Kr}$ in ${ }^{N a t} \mathrm{Kr}$ has been measured as $2 \cdot 10^{-11}$ [14]. In PandaX-II, a $6.6 \pm 2.2 \mathrm{ppt}$ of ${ }^{N a t} \mathrm{Kr}$ in xenon (mole/mole) had been achieved. In PandaX-4T, a new online distillation tower will be constructed which is capable of removing ${ }^{N a t} \mathrm{Kr}$ level to $0.1 \mathrm{ppt}$. The corresponding ER background in the low energy region is estimated as $0.0053 \mathrm{mDRU}$. A $20 \%$ uncertainty is adopted due to the uncertainty in the shape of the $\beta$ spectrum at low energies [15].

The contamination of dispersed ${ }^{222} \mathrm{Rn}$ in the liquid xenon is given particular attention. The ${ }^{222} \mathrm{Rn}$ level measured in the latest run of the PandaX-II is approximately $8 \mu \mathrm{Bq} / \mathrm{kg}$ [10, 11] using delayed coincidence between daughter ${ }^{214} \mathrm{Bi}^{2}{ }^{214} \mathrm{Po}$. Most ${ }^{222} \mathrm{Rn}$ atoms in the detector come from the decay chain of ${ }^{238} \mathrm{U}$. ${ }^{222} \mathrm{Rn}$ can emanate into target xenon through xenon plumbing, the cryostat and detector components. As the half-life (3.8 days) is relatively long, the distribution of ${ }^{222} \mathrm{Rn}$ is homogeneous in the target. PandaX-4T is performing emanation measurement to screen the materials, as well as implementing distillation and activated carbon filtration systems. Our aim is to control the ${ }^{222} \mathrm{Rn}$ level in xenon to within $1 \mu \mathrm{Bq} / \mathrm{kg}$. In the decay chain from ${ }^{222} \mathrm{Rn}$ to the stable ${ }^{210} \mathrm{~Pb}$, the $\beta$-decay of ${ }^{214} \mathrm{~Pb}$ dominates the ER contribution. $1 \mu \mathrm{Bq} / \mathrm{kg}$ translates to an ER background of $0.0114 \mathrm{mDRU}$ in the fiducial volume with a $10 \%$ systematic uncertainty of tagging ${ }^{222} \mathrm{Rn}$ using ${ }^{214} \mathrm{Bi}^{-214}{ }^{21} \mathrm{Po}$ coincidence.

The noble gas ${ }^{220} \mathrm{Rn}$ has a lower ability to emanate into the liquid xenon sensitive volume due to its shorter half-life. The results from the PandaX-II experiment (see [10, 11]) show that the background contribution from ${ }^{220} \mathrm{Rn}$ is small and is omitted here.

${ }^{136} \mathrm{Xe}$ is a two-neutrino double-beta decay isotope of xenon with a half-life of $2.17 \cdot 10^{21}$ years. The concentration of ${ }^{136} \mathrm{Xe}$ in natural xenon is approximately $8.9 \%$. In the simulation, the double-beta decay energy spectrum is taken from the DECAY0 code [16] and the average ER background rate in the low energy region is found to be $0.0023 \mathrm{mDRU}$. A $15 \%$ systematic uncertainty is assumed, according to the discussions in [17. The large amount of ${ }^{136} \mathrm{Xe}$ in the sensitive volume makes the PandaX-4T detector also sensitive to the search of neutrinoless double-beta decay [18]. 
Electron neutrinos, $\nu_{e}$, from solar nuclear reactions, contribute to the ER background at low energy by scattering elastically off electrons in the liquid xenon. The dominant flux component (92\%) of solar neutrinos is produced through the proton-proton fusion with a neutrino energy up to $400 \mathrm{keV}$. An electron capture reaction on ${ }^{7} \mathrm{Be}$ is the second largest flux component $(7 \%)$ in the low energy region, producing two mono-energetic neutrino lines at 384.3 and $861.3 \mathrm{keV}$. The pep and other sources contribute to the remaining $1 \%$. In the simulation, we take into account the neutrino oscillation and the reduced cross section for $\nu_{\mu, \tau}$. Simulations show that the solar neutrinos contribute an ER background of $0.009 \mathrm{mDRU}$, with a $2 \%$ uncertainty covering the systematic uncertainties of the neutrino flux and oscillation.

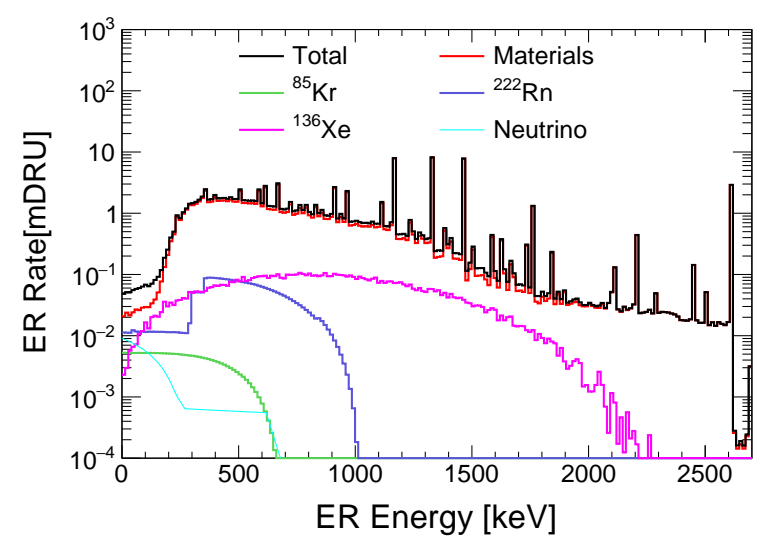

FIG. 6: The ER background from detector materials, intrinsic contaminations and neutrinos. The bold black line is the total background.

Including all sources mentioned above, the full ER background spectrum is shown in Figure 6. Based on the PandaX-II analysis, we assume that such a background could be mostly rejected using the $S 2 / S 1$ ratio with a $99.75 \%$ efficiency with $40 \%$ NR acceptance.

\section{NR Background}

The NR background is produced by neutron background via elastic scattering off the xenon nuclei. As it is difficult to differentiate it from the WIMP-nucleus scattering, it is crucial to control the neutron background.

The cosmogenic neutrons at CJPL has been suppressed to a negligible level. Instead, the neutron background comes from primordial decay of chains of ${ }^{238} \mathrm{U},{ }^{235} \mathrm{U}$ and ${ }^{232} \mathrm{Th}$ through the spontaneous fission (S.F.) and through $(\alpha, \mathrm{n})$ reactions. The neutron yield from the $(\alpha, \mathrm{n})$ reaction depends on the the $\alpha$ rate in the primordial decay chains and the target material. Common neutron producing targets include ${ }^{13} \mathrm{C},{ }^{14} \mathrm{~N},{ }^{17} \mathrm{O},{ }^{18} \mathrm{O},{ }^{19} \mathrm{~F}$, etc. For PTFE, the neutron generation rate is high because of the abundance of ${ }^{19} \mathrm{~F}$. For the neutron yield in PMTs, the ceramic stem $\left(\mathrm{Al}_{2} \mathrm{O}_{3}\right)$, the quartz window and the SS casing are simulated separately. For heavy nuclei such as copper, the neutron generation is mainly dominated by the S.F. from ${ }^{238} \mathrm{U}$.

With SOURCES4A [19] package, we calculate the neutron generation rates and energy spectra from S.F. and $(\alpha, n)$ mechanics in various materials, as shown in Figure 7. In all the materials, neutrons from the S.F. are dominated by the isotope of ${ }^{238} \mathrm{U}(>99 \%)$. However, such a process emits more than one neutron or $\gamma$, which causes multiscattering and can be rejected in the selection 20. A similar effect exists in the $(\alpha, n)$ reaction, that significant fraction of neutrons are emitted together with $\gamma \mathrm{s}$, which may also be rejected. Such suppression is included in the Geant4 simulation below, but details will be described elsewhere [21.

Using the BambooMC, we generate neutrons by sampling neutron energy spectrum from SOURCE4A in the corresponding material, in combination with $\gamma$ rays in the given $(\alpha, n)$ reaction when appropriate. We generate $10^{6}$ neutrons from each component and simulate the energy deposition inside the liquid xenon. The obtained NR energy spectrum and events distribution in the target are shown in Figures 8 and 9 . The so-called Lindhard factor is used in converting the NR energy into electron equivalent energy [22] when applying the energy selection window. The simulation shows that the veto compartment can reject approximately $50 \%$ of the NR background from the TPC materials.

To calculate the detected rate of NR events, we apply the detection efficiency as a funtion of NR energy obtained in the PandaX-II experiment, as shown in Figure 10. Within the WIMP search window, we predict the NR background 

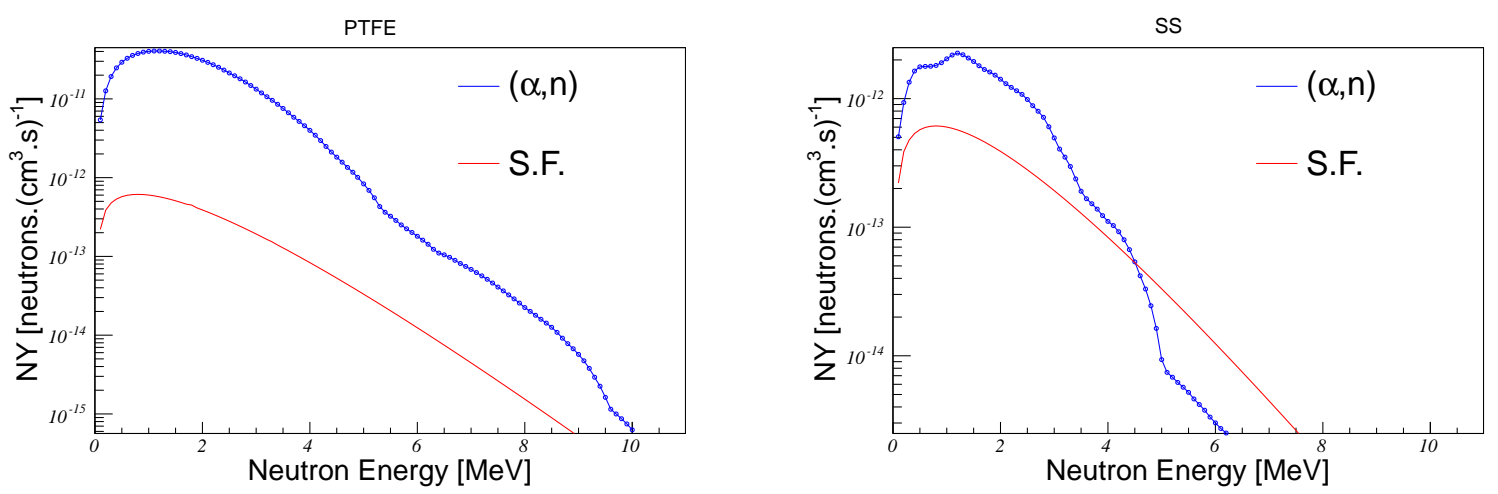

FIG. 7: Neutron Yield: S.F. vs $(\alpha, n)$. Top: PTFE. Bottom: Stainless steel. It shows the fission dominates the neutron generation for stainless steel, while $(\alpha, n)$ mechanics dominates the PTFE.

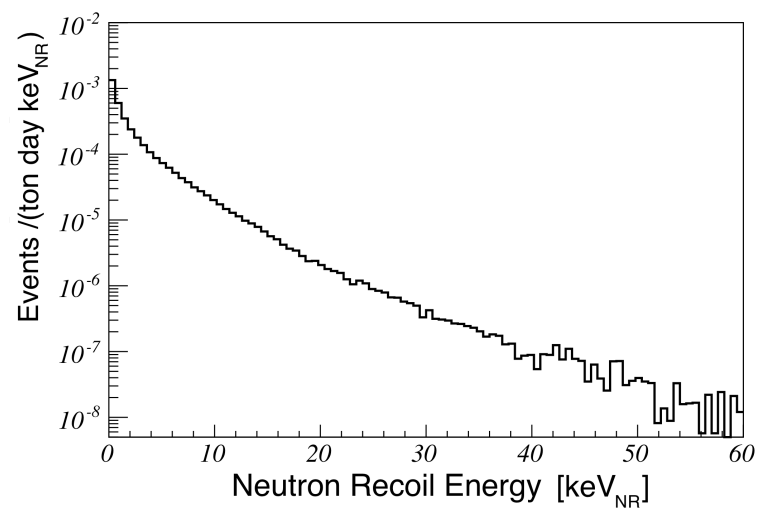

FIG. 8: The NR background spectrum from simulation.

from materials to be $2.0 \cdot 10^{-4} \mathrm{mDRU}$, with detailed values from different components shown in Table III. A $17 \%$ systematic uncertainty is adopted mainly from the neutron yield from the SOURCES4A.

In addition to the radioactive isotopes, neutrinos can also generate NR events through the coherent neutrino-nucleus scattering process (CNNS), a process which was recently observed using accelerator neutrinos by the COHERENT Collaboration [23. The nuclear recoil spectra from various neutrino sources are shown in Figure 11. For the dark matter candidate selection energy range, the dominant contribution is from the ${ }^{8} \mathrm{~B}$ and hep neutrinos. Based on the PandaX-II efficiency, we estimate that the neutrino CNNS contribution is $7.6 \cdot 10^{-5} \mathrm{mDRU}$ with $50 \%$ systematic

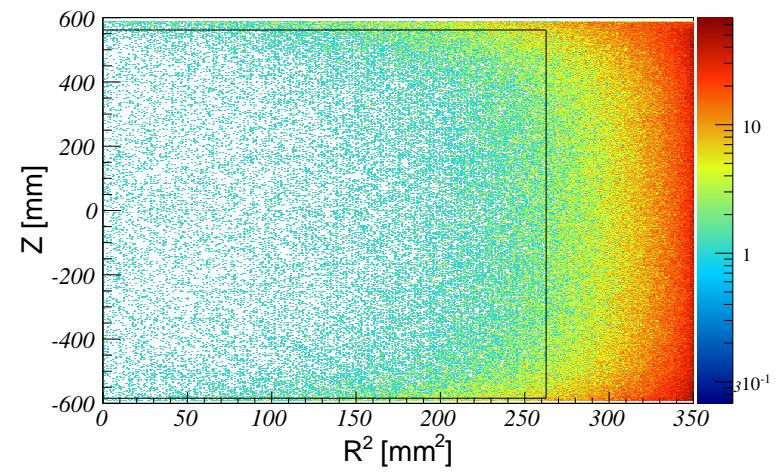

FIG. 9: Distribution of NR background events in the target from simulation. 


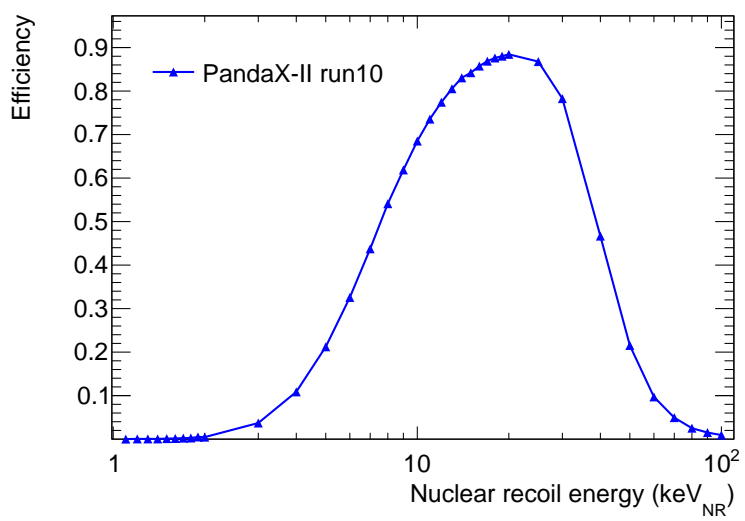

FIG. 10: NR events selection efficiency as a function of nuclear recoil energy in various runs of the PandaX-II.

TABLE III: NR background from detector materials for $E_{e e}$ within $(1 \mathrm{keV}, 10 \mathrm{keV})$.

\begin{tabular}{cc}
\hline \hline \multicolumn{2}{c}{ NR Background from materials } \\
\hline Source & NR rate in mDRU \\
\hline Inner vessel & $4.6 \pm 0.8 \cdot 10^{-5}$ \\
\hline Outer vessel & $5.3 \pm 0.9 \cdot 10^{-5}$ \\
\hline PMT Casing & $3.5 \pm 0.6 \cdot 10^{-5}$ \\
\hline PMT Window & $4.7 \pm 0.8 \cdot 10^{-6}$ \\
\hline PMT Stem & $3.9 \pm 0.7 \cdot 10^{-5}$ \\
\hline PTFE & $1.3 \pm 0.2 \cdot 10^{-5}$ \\
\hline Copper & $5.9 \pm 1.0 \cdot 10^{-6}$ \\
\hline Total material & $2.0 \pm 0.3 \cdot 10^{-4}$ \\
\hline
\end{tabular}

uncertainty due to selection efficiency and neutrino fluxes.

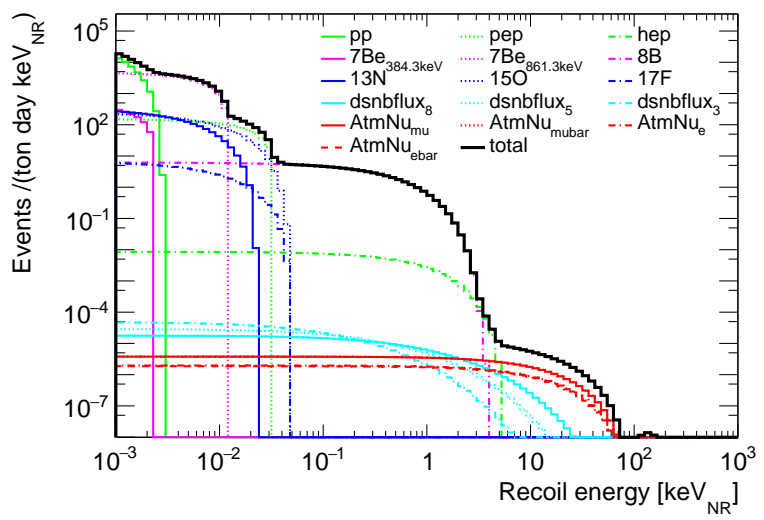

FIG. 11: Event rate from coherent neutrino-nucleus scattering in xenon. The neutrio sources include solar neutrinos $\left({ }^{8} \mathrm{~B}\right.$, hep,

${ }^{7} \mathrm{Be}$, CNO neutrinos, $p p$ chains), atmospheric neutrinos $\left(\nu_{e}, \bar{\nu}_{e}, \nu_{\mu}, \bar{\nu}_{\mu}\right)$ and diffuse supernovae $\left(\mathrm{T}_{\nu_{e}}=3 \mathrm{MeV}, \mathrm{T}_{\bar{\nu}_{e}}=5 \mathrm{MeV}\right.$, $\left.\mathrm{T}_{\nu_{x}}=8 \mathrm{MeV}\right)$.

\section{The PandaX-4T Background Summary}

As shown in Figure 3 and Figure 9, the ER and NR background events from the materials are concentrated close to the edge of the target. A fiducial cut is chosen to suppress background and to maintain a large effective mass: $35 \mathrm{~mm}$ below the gate electrode, $10 \mathrm{~mm}$ above the cathode, and within $512.5 \mathrm{~mm}$ in the radial direction. The total amount of xenon within this fiducial cut is 2.8 tons. Within this cut, the summary of ER and NR background is given 
in Table IV In the final background numbers, we have also assumed that the final ER rejection efficiency is $99.75 \%$ with $40 \%$ NR acceptance. After a two-year exposure, the final expected background is 2.5 (ER) and 2.3 (NR) events.

TABLE IV: Final background budget within the WIMP search window.

\begin{tabular}{ccc}
\hline \hline \multicolumn{2}{c}{ Summary of ER and NR backgrounds } \\
\hline Source & ER in mDRU & NR in mDRU \\
\hline Materials & $0.0210 \pm 0.0042$ & $2.0 \pm 0.3 \cdot 10^{-4}$ \\
\hline${ }^{222} \mathrm{Rn}$ & $0.0114 \pm 0.0012$ & - \\
\hline${ }^{85} \mathrm{Kr}$ & $0.0053 \pm 0.0011$ & - \\
\hline${ }^{136} \mathrm{Xe}$ & $0.0023 \pm 0.0003$ & - \\
\hline Neutrino & $0.0090 \pm 0.0002$ & $0.8 \pm 0.4 \cdot 10^{-4}$ \\
\hline Sum & $0.049 \pm 0.005$ & $2.8 \pm 0.5 \cdot 10^{-4}$ \\
\hline 2-year yield (evts) & $1001.6 \pm 102.2$ & $5.7 \pm 1.0$ \\
\hline after selection (evts) & $2.5 \pm 0.3$ & $2.3 \pm 0.4$ \\
\hline
\end{tabular}

\section{PHYSICS REACH OF THE PANDAX-4T}

Given a WIMP mass and WIMP-nucleon scattering cross section, the NR rate and spectrum are calculated using identical formalism as in Refs. [1]. The NR efficiencies in PandaX-II (Figure 10) is adopted for the WIMP NR events as well. For simplicity, we assume simple counting experiment with the so-called CLs method [24].

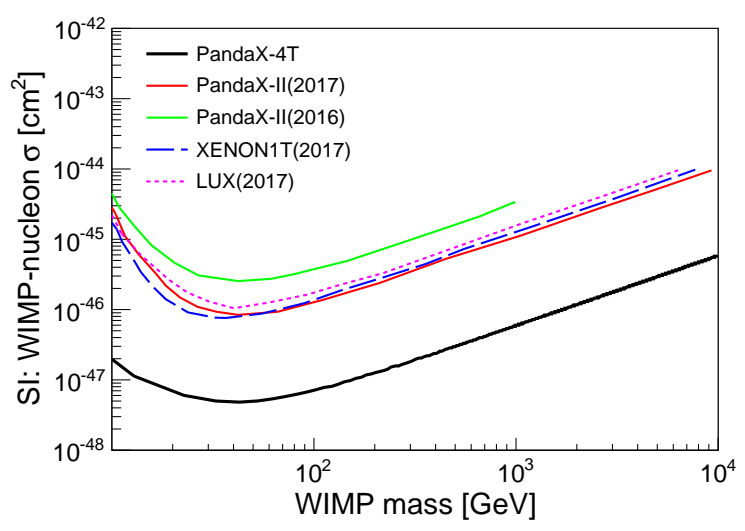

FIG. 12: PandaX-4T sensitivity (at 90\% C.L.) to WIMP-nucleon recoil events with an exposure of 5.6 ton-year. We compare the result with other leading experiments: LUX [8], XENON1T [9], PandaX-II [10, 11].

Under the expected background in Table IV the 90\% C.L. WIMP median sensitivity corresponds to a signal of 5 events after selection. The sensitivities for WIMP-nucleon spin-independent and spin-dependent interactions are shown in Figure 12 and Figure 13 , respectively, with a 5.6-ton-year exposure. For a WIMP mass of $m_{\chi}=40 \mathrm{GeV} / \mathrm{c}^{2}$, the sensitivity on the interaction cross section reaches a minimum at $6 \cdot 10^{-48} \mathrm{~cm}^{2}$ for spin-independent interaction. For the spin-dependent interaction, the strongest sensitivity reaches $9 \cdot 10^{-43} \mathrm{~cm}^{2}$ for the WIMP-neutron-only coupling and $3 \cdot 10^{-41} \mathrm{~cm}^{2}$ for the WIMP-proton-only coupling.

\section{CONCLUSION}

PandaX-4T is a next generation dark matter direct detection experiment with a multi-ton dual phase liquid xenon detector. In this paper we present a comprehensive simulation study of the background from radioactivity in the materials, intrinsic contaminations in the liquid xenon and neutrinos through. The WIMP candidate selection is chosen to be between $1 \mathrm{keV}$ and $10 \mathrm{keV}$ electron equivalent energy, single scattering in anti-coincidence with the veto compartment, and a vertex located in a 2.8-ton fiducial volume. In the NR signal region (with $S 2 / S 1$ cut), we estimate the background to be $2.5 \pm 0.3 \mathrm{ER}$ events and $2.3 \pm 0.4 \mathrm{NR}$ events for an exposure of 5.6 ton-year. The expected WIMP 

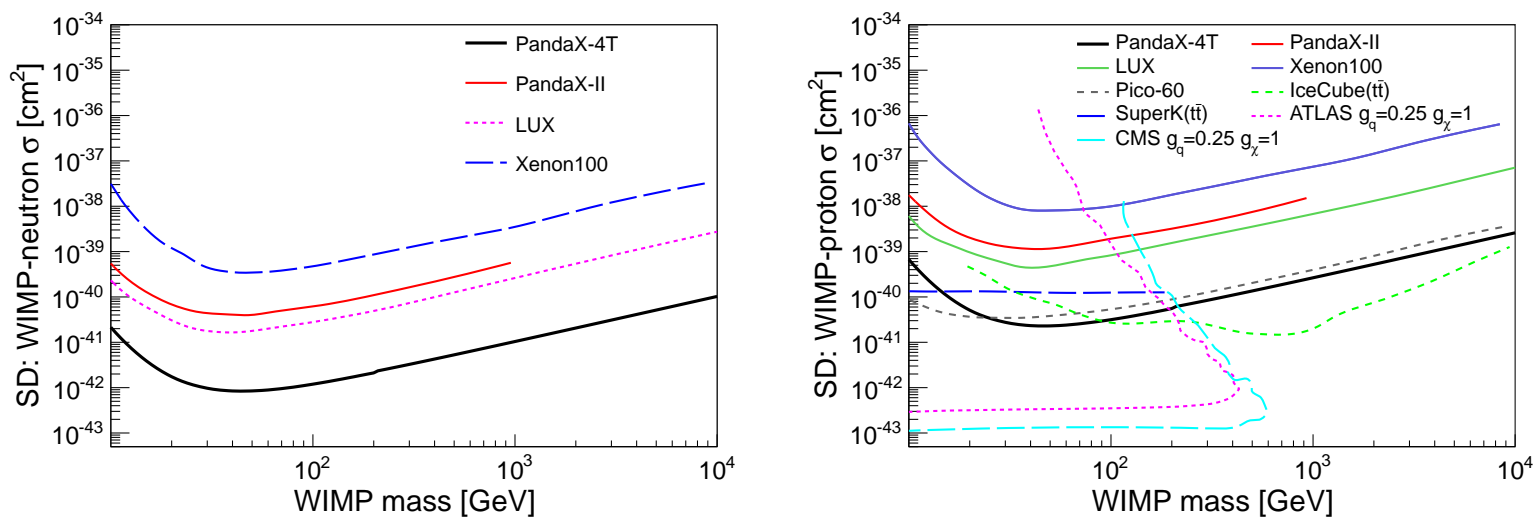

FIG. 13: PandaX-4T sensitivity (at 90\% C.L.) to WIMP-nucleon recoil for spin-dependent interaction, upper: proton-only, lower: neutron-only. We compare the results with the world data: LUX [25, XENON100 26], PandaX-II [27, CMS [28, ATLAS [29, PICO [30, 31, IceCube 32] and Super-K [33.

sensitivity of PandaX-4T can be more than a factor of ten improved from the currently running PandaX-II experiment.

This project has been supported by a 985-III grant from Shanghai Jiao Tong University, grants from National Science Foundation of China (Nos. 11435008, 11455001, 11505112, 11525522 and 11755001), and a grant from the Ministry of Science and Technology of China (No. 2016YFA0400301). We thank the support of grants from the Office of Science and Technology, Shanghai Municipal Government (No. 11DZ2260700, No. 16DZ2260200), and the support from the Key Laboratory for Particle Physics, Astrophysics and Cosmology, Ministry of Education. This work is supported in part by the Chinese Academy of Sciences Center for Excellence in Particle Physics (CCEPP) and Hongwen Foundation in Hong Kong. We also would like to thank Dr. Xunhua Yuan and Chunfa Yao of China Iron and Steel Research Institute Group, and Taiyuan Iron and Steel (Group) Co. LTD for crucial help on low background stainless steel. Finally, we thank the following organizations for indispensable logistics and other supports: the CJPL administration and the Yalong River Hydropower Development Company Ltd..

* Spokesperson, Corresponding author: xdji@sjtu.edu.cn

† Corresponding author: nzhou@sjtu.edu.cn

[1] S. M. Faber and J. S. Gallagher, Ann. Rev. Astron. Astrophys. 17, 135 (1979)

[2] G.R. Blumenthal, S. Faber, J.R. Primack and M.J. Rees, Nature 311, 517-525 (1984)

[3] P. A. R. Ade et al. (Planck Collaboration), Astron. Astrophys. 594 (2016) A13

[4] L. M. Widrow, B. Pym, J. Dubinski, Astrophys. J. 679, 1239-1259 (2008)

[5] G. Bertone, D. Hooper and J. Silk, Phys. Rept. 405, 279 (2005)

[6] D. Bauer et al., FERMILAB-CONF-13-688-AE

[7] J. Liu, X. Chen, X. Ji, Nature Phys. 13, 212 (2017)

[8] D. S. Akerib et al. (LUX Collaboration), Phys. Rev. Lett. 118, 021303 (2017)

[9] E. Aprile et al. (XENON1T Collaboration), Phys. Rev. Lett. 119, 181301 (2017)

[10] A. Tan et al. (PandaX Collaboration), Phys. Rev. Lett. 117, 121303 (2016)

[11] X. Cui et al. (PandaX Collaboration), Phys. Rev. Lett. 119, 181302 (2017)

[12] E. Aprile et al. (XENON1T Collaboration), JCAP 04, 027 (2016)

13] S. Agostinelli et al. (GEANT4 Collaboration), Nucl. Instrum. Meth. A 506 (2003) 250-303

[14] X. Du, K. Bailey, Z.-T. Lu, P. Mueller, T.P. O'Connor and L. Young, Rev. Sci. Instr. 75, 3223-3232 (2004)

[15] M. Selvi, in LOW RADIOACTIVITY TECHNIQUES 2013 (LRT 2013): Proceedings of the IV International Workshop in Low Radioactivity Techniques, vol. 1549, pp. 213-218, AIP Publishing, (2013)

[16] O. Ponkratenko, V. Tretyak and Y. Zdesenko, Phys. Atom. Nucl. 63, 1282-1287 (2000)

[17] J. Kotila and F. Iachello, Phys. Rev. C 85, 034316 (2012)

[18] X. Chen et al., Sci. China-Phys. Mech. Astron. 60, 061011 (2017)

[19] W. Wlison et al., Tech. Rep., LA-13639-MS, Los Almos, 1999.

[20] S. Shaw, Ph.D. thesis, University of College London (2016)

[21] Q. Wang et al. (PandaX Collaboration), in preparation (2018) 
[22] J. Lindhard el al., Mat. Fys. Medd. Dan. Vid. Selsk., 33, no. 10 (1963)

[23] D. Akimov et al. (COHERENT Collaboration), Science Vol. 357, Issue 6356, 1123-1126 (2017)

[24] A. L. Read, J. Phys. G 28 (2002) 2693

[25] D. S. Akerib et al. (LUX Collaboration), Phys. Rev. Lett. 116, 161302 (2016)

[26] E. Aprile et al. (XENON100 Collaboration), Phys. Rev. D 94, 122001 (2016)

[27] C. Fu et al. (PandaX Collaboration), Phys. Rev. Lett. 118, 071301 (2017)

[28] A. M. Sirunyan et al. (CMS Collaboration), Phys. Rev. D 97, 092005 (2018)

[29] M. Aaboud et al. (ATLAS Collaboration), J. High Energ. Phys. (2018) 2018:126

[30] C. Amole et al. (PICO Collaboration), Phys. Rev. D 93, 061101 (2016)

[31] C. Amole et al. (PICO Collaboration), Phys. Rev. D 93, 052014 (2016)

[32] M. G. Aartsen et al. (IceCube Collaboration), JCAP 1604, 022 (2016)

[33] K. Choi et al. (Super-Kamiokande Collaboration), Phys. Rev. Lett. 114, 141301 (2015) 